\title{
Gastroenteritis aguda por norovirus en pacientes con atención hospitalaria: estudio transversal
}

\author{
Ana P. Cervantes-Izaguirre ${ }^{1}$, Mario E. Rendón-Macías²*, Mónica Martina-Luna ${ }^{3}$, José Iglesias-Leboreiro³, \\ Isabel Bernárdez-Zapata ${ }^{3}$ y Araceli Martínez-Hernández ${ }^{1}$
}

${ }^{1}$ Facultad Mexicana de Medicina, Universidad La Salle; ${ }^{2}$ Escuela de Medicina Universidad Panamericana; Unidad de Investigación en Análisis y Síntesis de la Evidencia, Instituto Mexicano del Seguro Social; ${ }^{3}$ Departamento de Pediatría, Hospital Español de México. Ciudad de México, México

\begin{abstract}
Resumen
Introducción: Debido a la disponibilidad de técnicas moleculares en la atención clínica, las gastroenteritis agudas (GEA) por norovirus han retomado importancia como un agente causante de hospitalización. El objetivo de este estudio fue describir las características clínicas y evolutivas de pacientes menores de 16 años hospitalizados por GEA por norovirus. Métodos: Estudio retrospectivo. Se recabó información clínica de los pacientes atendidos en hospitalización del 1 de noviembre del 2016 al 28 de febrero del 2018 por GEA con detección de norovirus (genotipo I y II) en heces por medio de reacción en cadena de la polimerasa con transcriptasa inversa. Resultados: Estudiamos 103 pacientes; 96 (93.2\%; intervalo de confianza del 95\% [IC 95\%]: 86.6-96.7\%) con detección de genotipo II y 7 (6.8\%; IC 95\%: 5.3-8.7\%) de genotipo I; $76(73.8 \%) \leq 5$ años. El 48.5\% fueron atendidos durante el invierno. La evolución fue a la autolimitación en menos de 7 días en todos con manejo hidroelectrolítico. No hubo diferencias en la gravedad y síntomas según el grupo viral: en ambos predominaron los vómitos (82\%). Solo un paciente cursó con perforación intestinal por coinfección con Shigella sp.; tres pacientes (3.1\%) manifestaron crisis convulsivas (dos febriles y una epiléptica). Conclusiones: La GEA por norovirus, a pesar de causar una enfermedad meritoria de hospitalización, tiene un pronóstico favorable con autolimitación rápida. Su detección por pruebas rápidas en heces podría evitar la prescripción injustificada de antibióticos.
\end{abstract}

Palabras clave: Gastroenteritis aguda. Norovirus. Hospitalización. Antibióticos.

\section{Acute gastroenteritis due to norovirus in patients with hospital care: a cross-sectional study}

\section{Abstract}

Background: Because of the availability of molecular techniques in clinical care, acute gastroenteritis (AGE) due to norovirus has returned to importance as a causative agent of hospitalization. The aim of this study was to describe the clinical features and evolution of patients less than 16 years hospitalized for AGE associated with norovirus. Methods: Retrospective study. Clinical information of the patients attended from November 1, 2016 to February 28, 2018 by AGE with detection of norovirus (genotype I and II) in faeces by means of polymerase chain reaction with reverse transcriptase was collected. Results: We studied 103 patients; 96 (93.2\%; 95\% confidence interval [95\% Cl]: 86.6-96.7\%) with genotype Il detection and seven (6.8\%;

Correspondencia:

*Mario Enrique Rendón Macías E-mail: drmariorendon@gmail.com
Disponible en internet: 05-07-2019 Bol Med Hosp Infant Mex. 2019;76:176-181 www.bmhim.com 
95\% Cl: 5.3-8.7\%) genotype l; $76(73.8 \%) \leq 5$ years. $48.5 \%$ attended during the winter. The evolution was to self-limitation in less than 7 days in all with hydro electrolytic management. There were no differences in the severity and symptoms according to the viral group; in both cases the vomiting predominated (82\%). Only one patient had intestinal perforation due to co-infection with Shigella sp.; three patients (3.1\%) manifested seizures (two febrile and one epileptic convulsions). Conclusions: Despite causing a meritorious disease of hospitalization, GEA by norovirus has a favorable prognosis with rapid self-limitation. Its timely detection by rapid tests in feces could avoid the unjustified prescription of antibiotics.

Key words: Acute gastroenteritis. Norovirus. Hospitalization. Antibiotics.

\section{Introducción}

La gastroenteritis aguda (GEA) es un importante problema de salud en todo el mundo'. Constituye un motivo frecuente de consulta y hospitalización en la infancia, cuya principal etiología es la forma infecciosa². Alrededor del $90 \%$ de los casos infecciosos son causados por un virus, principalmente del género rotavirus, norovirus y adenovirus ${ }^{3-6}$. El rotavirus fue la principal causa de GEA en niños ${ }^{7,8}$ hasta la aparición de las vacunas antirrotavirus. Actualmente, el surgimiento de técnicas moleculares disponibles en la clínica para la búsqueda de otros virus ha permitido la detección de otros patógenos importantes en las diarreas, como el norovirus $3,4,9$.

Anteriormente conocido como virus Norwalk, el norovirus es un ARN virus de la familia Caliciviridae, con dos principales genotipos, GI y Gll, asociados con GEA en humanos ( 15 y $85 \%$, respectivamente). Su propagación es fácil por su resistencia a varios agentes antisépticos y el hecho de que necesita solamente 100 virones para causar una infección ${ }^{10,11}$. La predisposición a la infección se ha relacionado con la interacción de estos virus con los antígenos de grupos sanguíneos humanos ${ }^{12}$.

La infección por norovirus es común en México y en el mundo: se ha observado la presencia de anticuerpos antinorovirus hasta en el $85 \%$ de los niños de más de 2 años $^{12}$. En particular, durante el verano, este virus se ha detectado en el $30 \%$ de las heces de niños asintomáticos ${ }^{13}$.

La GEA por norovirus suele presentarse con evacuaciones líquidas no sanguinolentas, acompañadas de náusea, vómitos y dolor abdominal en un tiempo de 4 a 6 días. Suelen presentarse episodios autolimitados con manejo ambulatorio ${ }^{8,10}$, aunque, en ocasiones, se requiere el manejo hospitalario ${ }^{4,14}$. La tasa anual estimada en el mundo de internamiento hospitalario por esta causa es de 10 a 63.7 por cada 10,000 menores de 5 años de edad ${ }^{15-16}$ y de 7.9 por 10,000 niños de 5 a 19 años ${ }^{15}$.

Con la introducción de estudios moleculares para la detección de los distintos agentes enteropatógenos se facilitó el esclarecimiento del agente causal de la GEA. Además, es posible realizar una prescripción racional de antibióticos desde el servicio de emergencia ${ }^{10,14}$. En este trabajo se describen las características clínicas y evolutivas de los pacientes menores de 16 años hospitalizados por GEA por norovirus.

\section{Métodos}

Se llevó a cabo un estudio retrospectivo en un hospital escuela pediátrico privado de la Ciudad de México que cuenta con 41 camas de hospitalización censables, dos terapias intensivas (neonatal y pediátrica) y un servicio de urgencias con ocho camas censables. La atención en urgencias es de 5,198 pacientes al año, en promedio, con estancias no mayores a 6 horas.

Para cumplir con el objetivo, se revisaron los pacientes hospitalizados por GEA, definida como tres o más deposiciones de consistencia más blanda de lo normal en 24 horas, acompañadas o no de vómitos y examen positivo a norovirus en heces, durante el periodo del 1 noviembre de 2016 al 28 febrero del 2018. Se excluyeron los pacientes en terapia intensiva desde su ingreso, inmunocomprometidos y con enfermedades intestinales crónicas.

Desde 2015, se realiza el estudio molecular bacteriológico y virológico fecal en el hospital donde se recabaron los datos. Los estudios coprológicos se realizan de acuerdo con las condiciones clínicas de los pacientes y, sobre todo, en los hospitalizados. De estos pacientes, se solicitan coprocultivo y estudios moleculares para virus y bacterias. Las muestras son tomadas por hisopado y procesadas inmediatamente en el hospital. En particular, la determinación de norovirus se realizó con la técnica reacción en cadena de la polimerasa con transcriptasa inversa (Kit ARIES ${ }^{\circledR}$ Norovirus Assay Luminex), que determina los grupos I y II del virus ${ }^{17}$.

Se obtuvieron los siguientes datos de cada paciente: edad, sexo, días de evolución de la diarrea previos a la hospitalización, número de evacuaciones y consistencia, presencia de moco o sangre en heces, número de vómitos, fiebre y condición de hidratación. 
La gravedad al ingreso al hospital se determinó con la escala de Ruuska-Vesikari'18,19:

- Días de duración diarrea: 1-4 días = 1 punto; 5 días $=2$ puntos; $\geq 6=3$ puntos.

- Número máximo de evacuaciones diarreicas en 24 h: 1-3 deposiciones $=1$ punto; $4-5$ deposiciones $=2$ puntos; $\geq 6$ deposiciones $=3$ puntos.

- Días de duración de vómitos: 1 día = 1 punto; 2 días $=2$ puntos; $\geq 3$ días $=3$ puntos.

- Número máximo de vómitos en 24 h: 1 vómito $=1$ punto; 2 vómitos $=2$ puntos; $\geq 3$ vómitos $=3$ puntos.

- Fiebre: $37.1-38.4^{\circ} \mathrm{C}=1$ punto; $38.5-38.9^{\circ} \mathrm{C}=2$ puntos; $\geq 39^{\circ} \mathrm{C}=3$ puntos.

- Deshidratación: $1-5 \%=2$ puntos; $\geq 6 \%=3$ puntos.

- Tratamiento: rehidratación $=1$ punto; ingreso $=2$ puntos.

Se registraron las complicaciones clínicas.

El estudio fue aprobado por el comité de ética e investigación del hospital al cumplir con las normas internacionales de la Declaración de Helsinki y la Norma Nacional de Salud de México. Se consideró sin riesgo y se respetó la confidencialidad y anonimato de los pacientes.

\section{Análisis estadístico}

Los datos se analizaron por grupos de norovirus (NovGl o NovGlI). Las variables cualitativas se resumieron en frecuencias simples y porcentajes. Las diferencias entre los grupos se evaluaron utilizando la prueba de $\chi^{2}$ por máxima verosimilitud y la prueba exacta de Fisher de dos colas, según el número de datos. La diferencia entre los puntajes de gravedad se evaluó con la prueba no paramétrica $U$ de Mann Whitney. Los estimadores se presentan en las tablas con sus intervalos de confianza al 95\% (IC 95\%). Se utilizó el programa estadístico SPSS ${ }^{\circledR}$ versión 22. La significación estadística se fijó con un valor de $p<0.05$.

\section{Resultados}

Durante el periodo de estudio, 106 pacientes con determinación de norovirus en heces fueron hospitalizados. Tres de ellos se excluyeron del análisis: un preescolar con enfermedad oncológica, un lactante con síndrome de intestino corto y un lactante con sepsis grave meritoria de atención en terapia intensiva. Finalmente, 103 pacientes previamente sanos cumplieron con los criterios de inclusión de GEA por norovirus: 93.2\% del NovGII (IC 95\%: 86.6-96.7\%) y $6.8 \%$ del NovGI (IC 95\%: 5.3-8.7\%). En ambos grupos predominaron los pacientes de 2 a 5 años (45.6\%; IC 95\%: $36.3-55.2 \%$ ) y de sexo masculino (59.2\%; IC 95\%: 49.6-68.2\%), sin diferencias estadísticamente significativas entre grupos (Tabla 1). Solamente en el grupo con NovGll se encontraron antecedentes de prematuridad y gastrostomía (4.7\%; IC 95\%: 1.6-10.2\%).

Los casos con NovGl fueron más frecuentes durante el verano. Por el contrario, el grupo NovGll predominó en invierno $(p=0.06)$.

Se encontró una mediana de un día de evolución en los pacientes antes de ser atendidos en el hospital donde se realizó el estudio, sin diferencias entre los grupos. Solamente un paciente (NovGll) cursaba con una GEA de 6 días de evolución. Aunque no se evidenció una diferencia estadísticamente significativa con respecto a la intensidad de los síntomas, el grupo con NovGl se caracterizó por la presencia de vómitos y evaluaciones blandas a líquidas, mientras que en el grupo de NovGll predominaron los episodios con evaluaciones líquidas y moco. Siete pacientes $(6.8 \%)$ no presentaron de datos de deshidratación. No obstante, la mayoría fueron revisados por deshidratación moderada a severa $(59.2 \%)$, sin diferencias estadísticamente significativas entre los grupos de norovirus.

En la atención inicial, nueve pacientes con NovGll (9/96, 9.3\%; IC 95\%: 3.6-15.2\%) presentaron alguna complicación: ocho asociadas con la fiebre y la deshidratación con desequilibrio hidroelectrolítico y un paciente con una perforación intestinal meritoria de cirugía (Tabla 2). En un lactante con NovGl, además, se detectó rotavirus. Su evolución fue hacia la autolimitación, pero cursó con deshidratación severa. En el grupo NovGll, cuatro pacientes presentaron aislamiento de Campylobacter sp. (2 a 5 años de edad y en otoño) y otro, aislamiento de Shigella sp. Este último paciente fue un lactante de 8 meses con antecedente de ser prematuro, que presentó una perforación intestinal meritoria de manejo con antibióticos y resolución quirúrgica. Un escolar de 13 años también presentó coinfección con Escherchia coli (0157), con evolución a la autolimitación.

Todas las GEA se resolvieron antes de 8 días: la mediana fue de 3 días y el máximo de 7 días.

\section{Discusión}

Ante la disminución de pacientes hospitalizados por GEA causada por rotavirus $7,8,20,21$, es necesario informar sobre aquellas causadas por otros virus como norovirus. Aunque la mayoría de los casos de GEA no requieren de un manejo hospitalario, el manejo intrahospitalario se 
Tabla 1. Características de la infección por norovirus según tipo

\begin{tabular}{|c|c|c|c|c|c|c|c|}
\hline \multirow{2}{*}{$\begin{array}{l}\text { Datos } \\
\text { Edad } \\
\quad<2 \text { años } \\
2 \text { a } 5 \text { años } \\
6 \text { a } 11 \text { años } \\
12 \text { a } 16 \text { años }\end{array}$} & \multicolumn{2}{|c|}{ Norovirus $\mathrm{GI}(\mathbb{N}=7)$} & \multicolumn{2}{|c|}{ Norovirus GII (N = 96) } & \multicolumn{2}{|c|}{ Todos ( $N=103)$} & \multirow{2}{*}{$\begin{array}{c}\mathbf{p} \\
0.51^{*}\end{array}$} \\
\hline & $\begin{array}{l}\mathrm{n} \\
1 \\
4 \\
2 \\
0\end{array}$ & $\begin{array}{c}14.3 \% \\
57.1 \% \\
28.6 \% \\
0\end{array}$ & $\begin{array}{c}\mathrm{n} \\
28 \\
43 \\
17 \\
8\end{array}$ & $\begin{array}{c}29.2 \% \\
44.8 \% \\
17.7 \% \\
8.3 \%\end{array}$ & $\begin{array}{c}\mathrm{n} \\
29 \\
47 \\
19 \\
8\end{array}$ & $\begin{array}{c}28.2 \% \\
45.6 \% \\
18.4 \% \\
7.8 \%\end{array}$ & \\
\hline $\begin{array}{l}\text { Sexo } \\
\text { Masculino } \\
\text { Femenino }\end{array}$ & $\begin{array}{l}4 \\
3\end{array}$ & $\begin{array}{l}57.1 \% \\
42.9 \%\end{array}$ & $\begin{array}{l}57 \\
39\end{array}$ & $\begin{array}{l}59.4 \% \\
40.6 \%\end{array}$ & $\begin{array}{l}61 \\
42\end{array}$ & $\begin{array}{l}59.2 \% \\
40.8 \%\end{array}$ & $1.00^{\dagger}$ \\
\hline $\begin{array}{l}\text { Antecedentes } \\
\text { Pretérmino } \\
\text { Gastrostomía }\end{array}$ & $\begin{array}{l}0 \\
0\end{array}$ & & $\begin{array}{l}4 \\
1\end{array}$ & $\begin{array}{l}4.2 \% \\
1.0 \%\end{array}$ & $\begin{array}{l}4 \\
1\end{array}$ & $\begin{array}{l}3.9 \% \\
1.0 \%\end{array}$ & \\
\hline $\begin{array}{l}\text { Estación del año } \\
\text { Primavera (abril-junio) } \\
\text { Verano (julio-septiembre) } \\
\text { Otoño (octubre-diciembre) } \\
\text { Invierno (enero-marzo) }\end{array}$ & $\begin{array}{l}0 \\
3 \\
2 \\
2\end{array}$ & $\begin{array}{c}0 \\
42.9 \% \\
28.6 \% \\
28.6 \%\end{array}$ & $\begin{array}{c}16 \\
8 \\
24 \\
48\end{array}$ & $\begin{array}{c}21.9 \% \\
11.5 \% \\
25.0 \% \\
50 \%\end{array}$ & $\begin{array}{l}16 \\
11 \\
26 \\
50\end{array}$ & $\begin{array}{l}15.5 \% \\
10.7 \% \\
25.2 \% \\
48.5 \%\end{array}$ & $0.06^{*}$ \\
\hline Datos clínicos & & & & & & & \\
\hline $\begin{array}{l}\text { Días de evolución previos } \\
\text { Mediana } \\
\text { Percentil } 10 \\
\text { Percentil } 90\end{array}$ & $\begin{array}{l}1 \\
1 \\
2\end{array}$ & & $\begin{array}{c}1 \\
1 \\
4.3\end{array}$ & & $\begin{array}{l}1 \\
1 \\
3\end{array}$ & & $0.27^{\ddagger}$ \\
\hline $\begin{array}{l}\text { Evacuaciones } \\
\text { Blandas } \\
\text { Líquidas } \\
\text { Con moco } \\
\text { Moco y sangre }\end{array}$ & $\begin{array}{l}3 \\
1 \\
2 \\
1\end{array}$ & $\begin{array}{l}42.9 \% \\
14.3 \% \\
28.6 \% \\
14.3 \%\end{array}$ & $\begin{array}{c}16 \\
45 \\
32 \\
3\end{array}$ & $\begin{array}{c}16.7 \% \\
46.9 \% \\
33.3 \% \\
3.1 \%\end{array}$ & $\begin{array}{c}19 \\
46 \\
34 \\
4\end{array}$ & $\begin{array}{c}18.4 \% \\
44.7 \% \\
33.0 \% \\
3.9 \%\end{array}$ & $0.15^{*}$ \\
\hline $\begin{array}{l}\text { Vómitos } \\
\text { Presentes } \\
\text { Mediana eventos } \\
\text { Percentil } 10 \\
\text { Percentil } 90\end{array}$ & $\begin{array}{l}7 \\
3 \\
2 \\
5\end{array}$ & $100 \%$ & $\begin{array}{c}80 \\
4 \\
0 \\
8.3\end{array}$ & $83.3 \%$ & $\begin{array}{c}87 \\
4 \\
0 \\
8.6\end{array}$ & $82.0 \%$ & $0.95^{\ddagger}$ \\
\hline $\begin{array}{l}\text { Deshidratación } \\
\text { Ausente } \\
\text { Leve } \\
\text { Moderada } \\
\text { Severa }\end{array}$ & $\begin{array}{l}0 \\
4 \\
3 \\
0\end{array}$ & $\begin{array}{l}57.1 \% \\
42.9 \%\end{array}$ & $\begin{array}{c}7 \\
33 \\
54 \\
2\end{array}$ & $\begin{array}{c}7.3 \% \\
34.4 \% \\
56.2 \% \\
2.1 \%\end{array}$ & $\begin{array}{c}7 \\
37 \\
57 \\
2\end{array}$ & $\begin{array}{c}6.8 \% \\
35.9 \% \\
55.3 \% \\
1.9 \%\end{array}$ & $0.49 *$ \\
\hline $\begin{array}{l}\text { Gravedad } \\
\text { Moderada-severa } \\
\text { No deshidratado o leve } \\
\text { Media del puntaje (DT) }\end{array}$ & $\begin{array}{c}4 \\
3 \\
10\end{array}$ & $\begin{array}{c}57.1 \% \\
42.9 \% \\
(1.6)\end{array}$ & $\begin{array}{l}57 \\
39 \\
9.9\end{array}$ & $\begin{array}{c}59.4 \% \\
40.6 \% \\
(2)\end{array}$ & $\begin{array}{l}61 \\
42 \\
9.9\end{array}$ & $\begin{array}{l}59.2 \% \\
40.8 \% \\
(2)\end{array}$ & $1.00^{\ddagger}$ \\
\hline
\end{tabular}

* Prueba $\chi^{2}$ de máxima verosimilitud.

tPrueba exacta de Fisher dos colas.

‡Prueba U de Mann Whitney.

DT: desviación típica.

hace necesario en episodios con baja tolerancia a la rehidratación oral por vómitos o pérdida abundante de líquidos en evacuaciones. En estos casos, la disponibilidad de estudios moleculares virológicos permite al clínico establecer el agente causal y, con ello, normar mejor su conducta terapéutica. Los datos muestran que, en general, el curso de los pacientes con GEA por norovirus no es grave, tal como han informado otros estu$\operatorname{dios}^{22,23}$, aunque del 15 al $20 \%$ de ellos será hospitalizado por vómitos o deshidratación asociada, sobre todo con la diarrea ${ }^{3,4}$. La detección e información de casos de GEA hospitalizados por norovirus también contribuye a estimar la situación de esta enfermedad. Como se ha reportado en otros estudios ${ }^{4,24,25}$, se presentaron casos de GEA por norovirus durante todo el año, pero la frecuencia fue más elevada durante los meses del invierno. Esta información puede apoyar acciones preventivas específicas a futuro. 
Tabla 2. Complicaciones en los pacientes con infección por norovirus GII

\begin{tabular}{|l|c|c|}
\hline \multirow{2}{*}{ Datos } & \multicolumn{2}{|c|}{ Norovirus GII (N = 96) } \\
\cline { 2 - 3 } & $\mathbf{n}$ & $\%$ \\
\hline Crisis febril & 2 & 2.1 \\
\hline Íleo metabólico & 2 & 2.1 \\
\hline Acidosis metabólica & 1 & 1.0 \\
\hline Hiponatremia & 1 & 1.0 \\
\hline Hipoglucemia & 1 & 1.0 \\
\hline Crisis convulsivas & 1 & 1.0 \\
\hline Perforación intestinal & 1 & 1.0 \\
\hline
\end{tabular}

Los pacientes presentaron una sintomatología muy activa, lo que motivó a sus padres a solicitar atención urgente y, por sus condiciones clínicas, requirieron asistencia hospitalaria. La principal indicación fue el manejo de la deshidratación con sus complicaciones hidroelectrolíticas. Solamente se presentó un caso de hipoglucemia, condición más informada por rotavirus en comparación con norovirus ${ }^{20}$. Dada la importancia del manejo de rehidratación en la justificación de la hospitalización, y debido a que es el principal motivo de las complicaciones, su resolución permitió una estancia corta en todos los casos, con excepción del paciente quirúrgico.

Con respecto a los grupos de norovirus, se encontró al grupo Gll como el más prevalente (93\%) durante todo el año. En este grupo, el subgrupo Gll4 ha sido el más frecuente en otras partes del mundo 10,13,21,25. Debido al diseño del estudio, no se establecieron los genotipos.

Estudios previos han intentado diferenciar el comportamiento clínico de las GEA por norovirus según el grupo viral. Al igual que en este estudio, la presencia de vómitos parece ser más frecuente en los GEA por NovGl ${ }^{23,26}$, aunque la diferencia no ha sido concluyente. La presencia de crisis convulsivas, sobre todo asociadas con fiebre, suele observarse en pacientes con cursos rápidos de la enfermedad con infecciones por NovGll. Sin embargo, el comportamiento clínico aún no ha facilitado la predicción del genotipo causante y, nuevamente, la evolución es hacia la autolimitación, independientemente del grupo y del genotipo viral ${ }^{10,26}$.

Como en el caso de cinco pacientes, la detección de más de un agente viral o de una bacteria es frecuente ${ }^{13}$. La asociación parece no influir en la gravedad de la enfermedad, pero sí puede relacionarse con alguna complicación. En el paciente con perforación intestinal, el aislamiento de Shigella sp. justificó el manejo con antibióticos y la resolución quirúrgica.

Se considera es justificable establecer la relación causal de una GEA por norovirus en pacientes con un manejo hospitalario. En concordancia con lo informado en otros estudios $3,14,15,22,27$, el comportamiento autolimitado de los casos de GEA por norovirus en pacientes hospitalizados debería limitar el uso de antibióticos, y con ello evitar el surgimiento de bacterias resistentes a los mismos. Además, sirve de apoyo al médico en el manejo de la angustia de los padres y la presión a su uso. Se requieren más estudios para apoyar esta hipótesis ${ }^{28}$.

La información de casos hospitalizados de GEA por norovirus y su comportamiento permite estimar el posible impacto de la introducción de una vacuna a futuro, la cual se encuentra actualmente en desarrollo $0^{10,19,29}$.

Varias limitantes en este trabajo deben considerarse. Las condiciones serán aplicables a centros similares a este, urbanos, y quizá una población con un nivel socioeconómico medio y alto. Los criterios de hospitalización pueden diferir en otros centros de hospitalización. El comportamiento en pacientes inmunocomprometidos y con enfermedades enterales crónicas debe ser estudiado de manera independiente. La disponibilidad y costo de la detección de agentes virales debe analizarse basándose en la inversión e impacto en poblaciones específicas.

Al momento, los presentes datos pueden utilizarse para estimar un comportamiento de las GEA por norovirus en niños menores de 16 años con un episodio agudo y riesgo de agravamiento.

Como conclusión, se puede decir que los episodios de GEA por norovirus que requirieron manejo hospitalario se presentaron predominantemente en pacientes menores de 5 años de edad, con una gravedad moderada, curso corto hacia la autolimitación y escasas complicaciones graves. La detección de norovirus podría disminuir el uso innecesario de antibióticos.

\section{Responsabilidades éticas}

Protección de personas y animales. Los autores declaran que para esta investigación no se han realizado experimentos en seres humanos ni en animales.

Confidencialidad de los datos. Los autores declaran que han seguido los protocolos de su centro de trabajo sobre la publicación de datos de pacientes. 
Derecho a la privacidad y consentimiento informado. Los autores declaran que en este artículo no aparecen datos de pacientes.

\section{Conflicto de intereses}

Los autores declaran no tener ningún conflicto de intereses.

\section{Financiamiento}

El financiamiento de este trabajo fue aportado por los autores.

\section{Bibliografía}

1. Naghavi M, Abajobir AA, Abbafati C, Abbas KM, Abd-Allah F, Abera SF et al. Global, regional, and national age-sex specific mortality for 264 causes of death, 1980-2016: a systematic analysis for the Global Burden of Disease Study 2016. Lancet. 2017:390:1151-210.

2. Guarino A, Ashkenazi S, Gendrel D, Lo Vecchio A, Shamir R, Szajewska H, European Society for Pediatric Gastroenterology, Hepatology, and Nutrition; European Society for Pediatric Infectious Diseases. European Society for Pediatric Gastroenterology, Hepatology, and Nutrition/European Society for Pediatric Infectious Diseases evidence-based guidelines for the management of acute gastroenteritis in children in Europe: update 2014. J Pediatr Gastroenterol Nutr. 2014:59:132-52.

3. Tran A, Talmud D, Lejeune B, Jovenin N, Renois F, Payan C, et al. Prevalence of rotavirus, adenovirus, norovirus, and astrovirus infections and coinfections among hospitalized children in northern France. J Clin Microbiol. 2010;48:1943-6.

4. Friesema IHM, Boer RF, Duizer E, Kortbeek LM, Notermans DW, Norbruis OF, et al. Etiology of acute gastroenteritis in children requiring hospitalization in the Netherlands. Eur $\mathrm{J}$ Clin Microbiol Infect Dis. 2012;31:405-15

5. Li CSY, Chan PKS, Tang JW. Prevalence of diarrhea viruses in hospitalized children in Hong Kong in 2008. J Med Virol. 2009;81:1903-11.

6. Ca Jacob NJ, Cohen MB. Update on diarrhea. Pediatr Rev. 2016;37: 313-22.

7. Hernández-Cortez C, Aguilera Arreola MG, Castro-Escarpulli G. Situación de las enfermedades gastrointestinales en México. Enf Inf Microbiol. 2011;31:137-51

8. Hassan F, Kanwar N, Harrison CJ, Halasa NB, Chappell JD, Englund JA, et al. Viral etiology of acute gastroenteritis in < 2-year-old US children in the post-rotavirus vaccine era. J Pediatr Infect Dis Soc [Internet]. 2018 [citado octubre 2018]. Disponible en: https://academic.oup.com/jpids/ advance-article/doi/10.1093/jpids/piy077/5090096

9. López-Medina E, Parra B, Dávalos DM, López P, Villamarín E, Pelaez M. Acute gastroenteritis in a pediatric population from Cali, Colombia in the post rotavirus vaccine era. Int J Infect Dis. 2018;73:52-9.

10. Bányai K, Estes MK, Martella V, Parashar UD. Viral gastroenteritis. Lancet. 2018;392:175-86.
11. Canales RA, Reynolds KA, Wilson AM, Fankem SLM, Weir MH, Rose JB, et al. Modeling the role of fomites in a norovirus outbreak. J Occup Environ Hyg. 2019;16:16-26.

12. Nordgren J, Svensson L. Genetic susceptibility to human norovirus infection: an update. Viruses. 2019;11:226.

13. Peasey AE, Ruiz-Palacios GM, Quigley M, Newsholme W, Martinez J, Rosales G, et al. Seroepidemiology and risk factors for sporadic norovirus/Mexico strain. J Infect Dis. 2004;189:2027-36.

14. Biscaro V, Piccinelli G, Gargiulo F, laniro G, Caruso A, Caccuri F, et al. Detection and molecular characterization of enteric viruses in children with acute gastroenteritis in Northern Italy. Infect Genet Evol. 2018; 60:35-41.

15. Burke RM, Shih S-M, Yen C, Huang Y-C, Parashar UD, Lopman BA, et al. Burden of severe norovirus disease in Taiwan, 2003-2013. Clin Infect Dis [Internet]. 2018; 67:1373-8. Disponible en: https://academic. oup.com/cid/advance-article/doi/10.1093/cid/ciy298/5033797.

16. Bierhoff M, Arvelo W, Estevez A, Bryan J, McCracken JP, López MR, et al. Incidence and clinical profile of norovirus disease in Guatemala, 2008-2013. Clin Infect Dis. 2018;67:430-6.

17. Luminex. Disponible en: www.luminexcorp.com/eu/aries-norovirus-assay.

18. Ruuska T, Vesikari T. Rotavirus disease in Finnish chidren: use of numerical scores for clinical severity of diarrhoeal episodes. Scand J Infect Dis. 1990;22:259-67.

19. García-Magán C, de Castro-López MJ, Llovo-Taboada J, Curros-Novo C, Puente-Puig M, Sánchez-Fauquier A, et al. Caracterización microbiológica de las gastroenteritis agudas virales atendidas en un servicio de pediatría en un área de alta cobertura vacunal frente a rotavirus. Enferm Infecc Microbiol Clin. 2014;32:246-9.

20. Bonkoungou IJO, Ouédraogo N, Tamini L, Teguera RK, Yaméogo P, Drabo MK, et al. Rotavirus and norovirus in children with severe diarrhea in Burkina Faso before rotavirus vaccine introduction. J Med Virol. 2018; 90:1453-60

21. Siafakas N, Zerva L, Hatzaki D, Lebessi E, Chronopoulou G, Paraskakis I, et al. Molecular epidemiology of noroviruses in children in South Greece, 2013-2015. J Med Virol. 2018;90:1703-11.

22. Biçer S, Cöl D, Küçük Ö, Erdağ GC, Giray T, Uğraş M, et al. Is there any difference between the symptomatology and clinical findings of viral agents causing dehydration? Minerva Pediatr. 2018;70:165-74.

23. Xie J, Nettel-Aguirre A, Lee BE, Chui L, Pang XL, Zhuo R, et al. Relationship between enteric pathogens and acute gastroenteritis disease severity: a prospective cohort study. Clin Microbiol Infect. 2019;25:454-61.

24. Esteves A, Nordgren J, Tavares C, Fortes F, Dimbu R, Saraiva N, et al. Genetic diversity of norovirus in children under 5 years of age with acute gastroenteritis from Angola. Epidemiol Infect. 2018;146:551-7.

25. Makhaola K, Moyo S, Lechiile K, Goldfarb DM, Kebaabetswe LP. Genetic and epidemiological analysis of norovirus from children with gastroenteritis in Botswana, 2013-2015. BMC Infect Dis. 2018;18:246.

26. Kim BR, Choi GE, Kim YO, Kim MJ, Song ES, Woo YJ. Incidence and characteristics of norovirus-associated benign convulsions with mild gastroenteritis, in comparison with rotavirus ones. Brain Dev. 2018:40:699-706.

27. Farsi M, Roodbari F, Nejati B, Arashkia A, Jalilvand S, Nateghian A, et al. Prevalence and genetic diversity of norovirus genogroup II in children less than 5 years of age with acute gastroenteritis in Tehran, Iran. Med Microbiol Immunol. 2018;207:201-10.

28. Hernández-Avendaño $Y$, Díaz-Madero S, Rendón-Macías ME, Iglesias-Leboreiro J, Bernárdez-Zapata I. Conducta terapéutica de los médicos ante el resultado de las pruebas de detección de patógenos en niños con diarrea aguda. Rev Mex Pediatr. 2018;85:5-10.

29. Mattison CP, Cardemil CV, Hall AJ. Progress on norovirus vaccine research: public health considerations and future directions. Expert Rev Vaccines. 2018;17:773-84. 